\title{
Honorários praticados por operadoras de planos odontológicos e pelo SUS em relação aos definidos pelo Conselho Federal de Odontologia
}

\section{Fees charged by dental insurance companies and SUS as compared to those defined by the Brazilian Federal Dental Council}

Orlando Saliba ${ }^{1}$, Bruno Cabus Góes ${ }^{2}$, Cléa Adas Saliba Garbin ${ }^{1}$, Renata Reis dos Santos ${ }^{3}$, Artênio José Isper Garbin $^{1}$

\section{RESUMO}

Objetivo: Investigou-se o repasse de honorário ao cirurgião-dentista ofertado pelas empresas operadoras de planos odontológicos por grupos de procedimentos odontológicos. Materiais e Métodos: Os valores praticados por operadoras de planos foram definidos a partir da média obtida entre valores das tabelas de cinco planos com abrangência nacional. Esses valores médios e os valores pagos pelo SUS foram comparados com os estabelecidos na tabela VRCC - CFO. Para a comparação, foram obtidos valores médios para os seguintes grupos de procedimentos: preventivos, de dentística, de endodontia e de cirurgia. Os valores dos procedimentos preventivos foram comparados com os demais grupos de procedimentos. Resultados: Os planos aplicaram um desconto médio de $54,51 \%$ sobre os preços sugeridos pelo CFO. O maior desconto foi observado no grupo de procedimentos preventivos (57,09\%, em média). Conclusão: As empresas operadoras de planos e convênios investigadas estão aplicando um desconto abusivo sob os preços sugeridos pelo CFO. É necessário identificar alternativas para que se possam reverter o quadro identificado por esse estudo.

Descritores: Honorários odontológicos. Seguro odontológico. Planos de sistemas de saúde.

\section{INTRODUÇÃO}

Os planos e seguros privados de saúde fazem parte de uma parcela importante do sistema de prestação de assistência à saúde no Brasil, denominada de assistência médica suplementar. Atualmente, estas empresas mantêm, sobre sua tutela, a saúde de mais de 42 milhões de brasileiros, equivalente a quase $25 \%$ da população nacional ${ }^{1}$, movimentando anualmente bilhões de reais.

O setor odontológico está atravessando uma fase paradoxal, caracterizada pelo excesso de profissionais no mercado, queda na demanda de pacientes, diminuição do poder aquisitivo e melhoria da saúde bucal da população, favorecendo o surgimento de uma nova modalidade de forma de trabalho para o cirurgião-dentista, os convênios e cooperativas odontológicas ${ }^{1-3}$. Muitos profissionais passaram a atender em seus consultórios particulares, pacientes associado a algum convênio ou cooperativa, e poucos relatam estar financeiramente realizados em sua profissão ${ }^{4}$. Entre as dificuldades relatadas na carreira pelos profissionais, a alta competitividade e a saturação de mercado são os principais motivos para aderir a esta nova modalidade de trabalho. Para os cirurgiões-dentistas, os convênios servem como uma forma de obter um maior número de paciente nas clínicas particulares, preenchendo os horários vagos $^{6-9}$.

Com o intuito de orientar a remuneração mínima dos cirurgiões dentistas, o CFO - Conselho Federal de Odontologia publicou no Diário Oficial da União - DOU tabelas de honorários com valores referenciais para procedimentos odontológicos ${ }^{10}$. Muitos convênios utilizam tabelas próprias para a remuneração dos seus credenciados, com honorários muitas vezes abaixo dos propostos pela

\footnotetext{
${ }^{1}$ Departamento de Odontologia Preventiva e Social, Faculdade de Odontologia de Araçatuba (FOA), Universidade Estadual Paulista Júlio Mesquita (UNESP), Araçatuba, SP, Brasil

${ }^{2}$ Cirurgião-dentista

${ }^{3}$ Programa de Pós-Graduação em Odontologia, Faculdade de Odontologia de Araçatuba (FOA), Universidade Estadual Paulista Júlio Mesquita (UNESP), Araçatuba, SP, Brasil

Contato: osaliba@foa.unesp.br, brunomcz@hotmail.com, cgarbin@foa.unesp.br, renatitars@yahoo.com.br, agarbin@foa.unesp.br
} 
Tabela Nacional de Convênios e Credenciamentos VRCC - CFO que define valores referenciais para a remuneração de honorários por procedimentos a serem pagos à profissionais credenciados por planos e convênios odontológicos ${ }^{11}$.

As várias formas de remuneração produzem distintos efeitos no comportamento do prestador de serviços de saúde, interferindo diretamente na gestão dos sistemas de assistência e na prática da Odontologia ${ }^{1}$. Diante ao exposto investigou-se o repasse de honorário ao cirurgião-dentista ofertado pelas empresas operadoras de planos odontológicos e pelo Sistema Único de Saúde (SUS) por grupos de procedimentos odontológicos em comparação com valores definidos na tabela de VRCC-CFO.

\section{MATERIAIS E MÉTODOS}

Foram coletadas tabelas de preços para remuneração de honorários profissionais aos cirurgiões-dentistas credenciados de cinco empresas operadoras de planos odontológicos com abrangência nacional, bem como a tabela operada pelo SUS.

Os valores de remuneração de honorários praticados pelas empresas operadoras e pelo SUS foram comparados com os da tabela e de VRCCCFO, em vigor, publicada no Diário Oficial da União, em $2002^{10}$. Os procedimentos foram agrupados em: procedimentos preventivos e procedimentos especializados (dentística, endodontia e cirurgia), conforme a classificação de procedimentos do
Sistema Único de Saúde - SUS, descrita no Código de procedimentos SIA/SUS ${ }^{12}$.

Para representar os valores praticados pelas operadoras de planos odontológicos, foram obtidas médias dos valores praticados por cinco operadoras. Após agrupamento dos procedimentos, os valores médios foram obtidos para cada grupo considerandose os valores das operadoras de plano, do SUS e da tabela VRCC-CFO. O percentual médio de desconto foi obtido pela comparação dos valores médios praticados pelos planos e pelo SUS com os definidos na tabela VRCC-CFO. O percentual de desconto nos procedimentos preventivos foi comparado aos demais grupos de procedimentos. Para realização dos cálculos das médias e porcentagens foi utilizado o software Excel (Office 2010).

\section{RESULTADOS}

Considerando-se todos os procedimentos em conjunto, as empresas operadoras de planos odontológicos aplicaram um desconto médio de $54,51 \%$ sobre os preços sugeridos na tabela VRCC - CFO (Tabela 1). As empresas operadoras de planos odontológicos aplicam o desconto médio de $57,09 \%$ sobre o valor dos procedimentos preventivos sugeridos na tabela VRCC - CFO, sendo este o grupo com maior desconto médio, seguido pelo grupo de procedimentos cirúrgicos $56,18 \%$, de dentística $53,69 \%$ e endodontia $53,07 \%$ (Tabela 1 ).

Tabela 1 - Descontos médios aplicados por empresas operadoras de planos odontológicos sobre a tabela VRCC - CFO, por grupo de procedimentos

\begin{tabular}{llll}
\hline PROCEDIMENTOS & CFO & MÉDIAS PLANOS & MÉDIAS DE DESCONTO (\%) \\
\hline Preventivos & $\mathrm{R} \$ 43,28$ & $\mathrm{R} \$ 18,57$ & 57,09 \\
Dentística & $\mathrm{R} \$ 54,86$ & $\mathrm{R} \$ 25,51$ & 53,69 \\
Endodontia & $\mathrm{R} \$ 109,49$ & $\mathrm{R} \$ 51,39$ & 53,07 \\
Cirurgia & $\mathrm{R} \$ 54,74$ & $\mathrm{R} \$ 23,99$ & 56,18 \\
Média Total & $\mathbf{R} \$ \mathbf{6 5 , 5 9}$ & $\mathbf{R} \$ \mathbf{2 9 , 8 4}$ & $\mathbf{5 4 , 5 1}$ \\
\hline
\end{tabular}

Os procedimentos especializados foram mais valorizados que os procedimentos preventivos tanto na tabela VCRR-CFO, como pelas operadoras de planos odontológicos e pelo SUS. A remuneração dos procedimentos cirúrgicos foi em média $20,93 \%$ maior do que dos procedimentos preventivos. Os planos e convênios também pagam mais pelos procedimentos cirúrgicos $(22,58 \%)$, enquanto que o SUS valoriza $(10,51 \%)$ os procedimentos cirúrgicos quando comparados aos procedimentos preventivos (Tabela 2). Em média, os planos e convênios pagaram $26,91 \%$ a mais por um procedimento de dentística quando comparado aos valores ofertados por procedimento preventivo; já o SUS aumentou essa discrepância pagando em média 37,66\% a mais para os procedimentos de dentística em relação aos procedimentos preventivos (Tabela 2). Os procedimentos endodônticos foram melhores remunerados $(63,86 \%)$ do que os procedimentos preventivos pelas empresas operadoras de planos e convênios, enquanto que o SUS pagou $32,11 \%$ a mais para realização de procedimentos endodônticos em relação aos valores pagos a procedimentos preventivos (Tabela 2). 
Tabela 2 - Diferença na remuneração entre grupos de procedimentos quando comparados ao grupo de procedimentos preventivos

\begin{tabular}{llll}
\hline PROCEDIMENTOS & PREVENTIVOS CFO (VRCC) (\%) & PREVENTIVOS PLANOS & PREVENTIVOS SUS \\
\hline Dentística & 21,11 & 26,91 & 37,66 \\
Endodontia & 60,47 & 63,86 & 32,11 \\
Cirurgia & 20,93 & 22,58 & 10,51 \\
\hline
\end{tabular}

\section{DISCUSSÃO}

Tentando recuperar o número de pacientes nas clínicas particulares, o cirurgião-dentista passou a firmar contratos de prestação de serviços através dos convênios e credenciamentos ${ }^{6}$. Nesse estudo, observou-se que os valores firmados entre as partes estão abaixo da tabela estabelecida pelo CFO.

Em estudo realizado por D'Avila ${ }^{2}$, os valores pagos pela tabela de honorários utilizada pelos sistemas de convênio foram classificados por $76,8 \%$ dos profissionais como regular ou ruim, e estes ainda mostraram descontentamento em relação ao prazo de pagamento e tratamento que recebem das empresas operadoras $^{2}$. Os cirurgiões-dentistas que trabalham vinculados a algum tipo de odontologia de grupo apresentam-se insatisfeitos ${ }^{2,9}$.

Esta insatisfação e a baixa remuneração podem acarretar em um tratamento inadequado no atendimento das necessidades do paciente, contribuindopara a massificaçãoe a despersonalização nas relações com eles ${ }^{14}$.

Observou-se uma valorização dos procedimentos clínicos em detrimento dos preventivos, mostrando a supervalorização da Odontologia curativa. Mesmo o serviço público de saúde que deveria incentivar as atividades de prevenção, determina valores inferiores aos preconizados pela tabela estipulada pelo CFO.

Os cirurgiões-dentistas que se submetem aos preços praticados pelas operadoras estão em desacordo com o Código de Ética Odontológico, que diz no seu Art. $13^{\circ}$ que: "O cirurgião-dentista deve evitar o aviltamento, ou submeter-se a tal situação inclusive por parte de convênios e credenciamentos, de valores dos serviços profissionais, não os fixando de forma irrisória ou inferior aos valores referenciais para procedimentos odontológicos"13.

O CFO através dos CROs deveria criar comissões especiais, regionalizadas, para avaliar os preços de honorários praticados por empresas operadoras de planos odontológicos, a fim de reduzir a discrepância entre os preços sugeridos na VRCC - CFO e os atualmente ofertados pelas empresas operadoras. Cabe ao CFO promover campanha de agregação da classe profissional, com o intuito de aumentar o poder de negociação frente às empresas operadoras de planos odontológicos a fim de coibir discrepâncias exageradas em relação à tabela VRCC - CFO. O CFO pode ainda dirigir campanha ao usuário final, paciente consumidor, divulgando uma classificação das empresas operadoras por remuneração de honorários aos prestadores. Estudos ainda devem ser realizados para verificar a ocorrência de direcionamento equivocado da prática odontológica para determinado grupo de procedimentos como resultado da interferência dos honorários na decisão dos profissionais por realizar diferentes tipos de procedimentos odontológicos, incluindo a adoção de práticas preventivas.

Por fim, deve-se considerar e deixar registrado que a tabela VRCC - CFO utilizada como base comparativa foi publicada no Diário Oficial da União em Janeiro de 2002, não sofrendo reajuste por 4 anos, necessitando de uma imediata reavaliação e um reajuste dos valores.

\section{CONCLUSÃO}

A remuneração por procedimentos odontológicos ofertados pelas empresas operadoras de planos odontológicos aos cirurgiões-dentistas fica muito aquém dos valores sugeridos na tabela VRCC-CFO. Os procedimentos preventivos são menos remunerados que os grupos de procedimentos especializados, tanto pelas operadoras de plano odontológico como pelo SUS.

\section{ABSTRACT}

Aim: The present study investigated the fees paid by dental insurance companies to dental surgeons, referrent to groups of dental procedures. Materials and Methods: The fees offered by dental insurance companies were defined according to the average values from the price tables of 5 insurance plans that offered national coverage. These average values and the values paid by the Brazilian Unified Health System (SUS) were compared with those set forth in the VRCC table from Brazilian Federal Dental Council (CFO). For comparison, average values were obtained for the following groups of procedures: preventive, aesthetic, endodontic, and 
surgical. The values of these preventive procedures were compared with other groups of procedures. Results: The plans applied an average discount of approximately $54.51 \%$ upon the prices suggested in the CFO price list. The highest discount was observed within the group of preventive procedures $(57.09 \%$, on average). Conclusion: The evaluated health insurance companies in vestigated in the present study are applying an abusive discount upon the prices suggested in the CFO price list. It's necessary to identify alternatives to reverse this situation.

Uniterms: Fees dental. Insurance dental. Health systems plans.

\section{REFERÊNCIAS}

1. Silveira LS. Prevenção de doenças e promoção da saúde: diferenciais estratégicos na conjuntura da saúde suplementar. Rio de Janeiro: Escola Nacional de Saúde Pública, Fundação Oswaldo Cruz; 2004.

2. D'avila S, Oliveira PAP, Lucas RSCC, Souza EA. Assistência odontológica x planos de saúde: um estudo em Campina Grande, Paraíba, Brasil. Pesqui Bras Odontopediatria Clín Integr. 2007; 7:259-63.

3. Simplício AHLS, Santos PA, Campos JADB. Perfil dos convênios odontológicos no município de Araraquara -SP, Brasil. Cienc Odontol Bras. 2008; 11: 30-9.

4. Bastos JRM, Aquilante AG, Almeida BS, Lauris JRP, Bijella VT. Análise do perfil profissional de cirurgiões dentistas graduados na Faculdade de Odontologia de Bauru - SP entre os anos de 1996 e 2000. J Appl Oral Sci. 2003; $11: 283-9$.

5. Sposati A, Lobo E. Controle social e políticas públicas. Cad Saúde Pública. 1992; 8:366-78.
6. Garcia PPNS, Cobra CS. Condições de trabalho e satisfação de cirurgiões-dentistas credenciados por convênios odontológicos. Rev Odontol UNESP. 2004; 33:115-22.

7. Belardinelli VH. Clínica particular, necessidade e possibilidade de fazê-la crescer: os convênios. Odontol Mod. 1987; 14:37-48.

8. Ferreira RA. O valor da saúde bucal nas empresas. Rev Assoc Paul Cir Dent. 1995; 49:96-107.

9. Serra MC, Henriques C. Participação de cirurgiões-dentistas em empresas de odontologia de grupo. Rev ABO Nac. 2000; 8:80-5.

10. Conselho Federal de Odontologia. Tabela de valores referenciais para convênios e credenciamentos. Diário Oficial da União. 2002 jan 24.

11. Lemos JA, Wanderley MS, Moreira PVL, Rabello PM, Caldas JR. AF, Rosenblatt A. Estudo comparativo entre convênios odontológicos, empresas e demais entidades prestadoras e/ou contratantes de serviços odontológicos na cidade de João Pessoa/PB. Rev Fac Odontol Pernamb. 1997; 15:73-6

12. Ministério da Saúde. Tabela de procedimentos do Sia-SUS. [acesso em 2005 jun 22]. Disponível em: http://www.saude.sc.gov.br/download/sia sih/sia/tabsia0406.zip.

13. Conselho Federal de Odontologia. Código de ética profissional. Florianópolis: CFO; 2003.

14. Fortes PAC. Ética e saúde: questões éticas, deontológicas e legais, tomada de decisões, autonomia e direitos do paciente, estudo de casos. São Paulo: EPU; 1998.

\author{
Autor correspondente \\ Renata Reis dos Santos \\ Rua José Bonifácio,1443 - Vila Mendonça \\ CEP:16011-050 - Araçatuba - SP - Brasil \\ E-mail: renatitars@yahoo.com.br
}

